\title{
Medial Occipital Lobe Hyperperfusion Identified by Arterial Spin-Labeling: A Poor Prognostic Sign in Patients with Hypoxic- Ischemic Encephalopathy
}

\author{
A. de Havenon, A. Sultan-Qurraie, D. Tirschwell, W. Cohen, J. Majersik, and J.B. Andre
}

\begin{abstract}
SUMMARY: Hypoxic-ischemic encephalopathy carries an uncertain prognosis. We sought to retrospectively assess the prognostic value of arterial spin-labeling MR imaging in 22 adult patients diagnosed with hypoxic-ischemic encephalopathy. Quantitative CBF maps were generated from the MO map, and arterial spin-labeling data on a per-voxel basis were regionally interrogated via visual inspection and ROI placement. Hyperperfusion was defined as regional increases in CBF of $>20 \%$ (relative to global CBF) and/or $>100 \mathrm{~mL} / 100 \mathrm{~g} / \mathrm{min}$. Eleven of 22 patients had prominent bilateral medial occipital lobe hyperperfusion, all of whom died before hospital discharge. One patient who had nondistinct arterial spin-labeling hyperperfusion and restricted diffusion survived. Medial occipital lobe hyperperfusion is a distinctive pattern that merits prospective investigation in a cohort of patients with moderate hypoxic-ischemic encephalopathy to determine its predictive ability in patients with a higher likelihood of survival.
\end{abstract}

ABBREVIATIONS: $\mathrm{ASL}=$ arterial spin-labeling; $\mathrm{HIE}=$ hypoxic-ischemic encephalopathy; $\mathrm{MOLH}=$ medial occipital lobe hyperperfusion

$\mathrm{H}^{2}$ ypoxic-ischemic encephalopathy (HIE) can result from cardiac arrest, profound hypotension, or respiratory distress secondary to a variety of medical causes, which share the final common pathway of neuronal death from failure of oxidative metabolism. ${ }^{1}$ Despite advances in its treatment, patients with HIE have high rates of morbidity and mortality. ${ }^{2}$ Given that the severity of HIE varies widely, a wealth of predictive prognostic tools exist, ranging from clinical markers, including level of consciousness and motor response to central noxious stimulation, to neurophysiologic testing, such as electroencephalogram, laboratory markers, and somatosensory-evoked potentials. Although traditionally difficult to perform in this typically clinically unstable population, MR imaging examination is the neuroimaging test of choice, despite the observation that functional recovery following extensive gray matter infarction can occur, suggesting that conventional MR imaging findings lack specificity. ${ }^{3}$

CBF is typically reduced in the first $24-48$ hours after HIE, but delayed cerebral hyperperfusion can develop thereafter, a finding

Received February 10, 2015; accepted after revision April 30.

From the Department of Neurology (A.d.H., J.M.), University of Utah, Salt Lake City, Utah; and Departments of Neurology (A.S.-Q., D.T.) and Radiology (W.C., J.B.A.), University of Washington, Seattle, Washington

Paper previously presented at: Annual Meeting of the Radiological Society of North America, November 30 to December 5, 2014; Chicago, Illinois.

Please address correspondence to Jalal B. Andre, MD, Department of Radiology, Harborview Medical Center, University of Washington, Box 357115, 1959 NE Pacific St, NW011, Seattle, WA 98195-7115; e-mail: drjalal@uw.edu

http://dx.doi.org/10.3174/ajnr.A4444 first observed with xenon-enhanced CT. ${ }^{4,5}$ This hyperperfusion appears to correlate with poor clinical outcome and was theorized to be the result of a loss of vascular resistance. Hyperperfusion after HIE has also been observed with arterial spin-labeling (ASL) MR imaging in both pediatric and adult patients, but in nonspecific patterns that have often corresponded with regions of restricted diffusion. ${ }^{6,7}$ ASL is a noninvasive MR imaging technique that produces perfusion maps that are comparable with contrastbased methods such as dynamic susceptibility contrast MR imaging. ${ }^{8}$ Because the brain and kidneys are both very sensitive to hypotension and lack of oxygen, HIE is often accompanied by transient acute renal injury, which can be a contraindication to gadolinium-based contrast agent administration in MR imaging. ${ }^{9,10}$ In light of this limitation, we sought to further clarify whether the presence of hyperperfusion, as previously reported in patients with $\mathrm{HIE}^{6}$ and detected with ASL, could be used as a marker of survival prognosis and to examine its relationship to standard prognostic tests.

\section{Case Series}

We performed a retrospective data base and patient chart review at an urban tertiary care academic hospital and included adult patients (18 years of age or older) with a discharge diagnosis of HIE. All patients had been admitted to our intensive care unit between September 2012 and January 2014 with a Glasgow Coma Scale score of $<8$. All patients underwent routine brain MR imaging on a 3T scanner (Tim Trio; Siemens, Erlangen, Germany) as part of standard clinical care. Acquired sequences varied slightly 


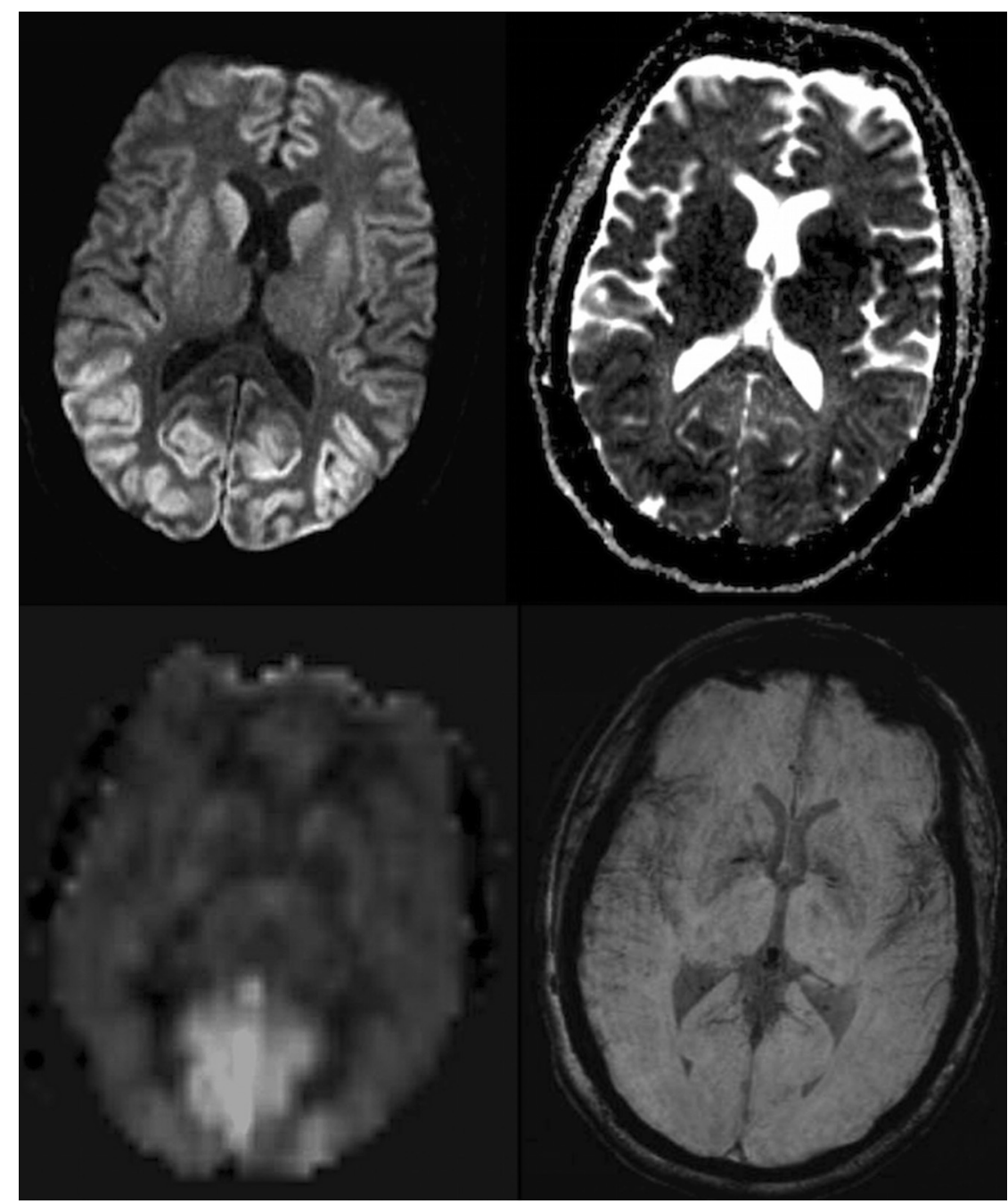

FIG 1. Brain MR imaging from a 44-year-old woman who had a ventricular fibrillation cardiac arrest 5 days prior. Top left: axial DWI with extensive parieto-occipital cortical and deep gray matter restricted diffusion. Top right: axial ADC map with a lesser amount of corresponding hypointensity from low ADC values, primarily located in the occipital cortex, likely secondary to early pseudonormalization. Bottom left: axial ASL CBF with prominent hyperperfusion of the bilateral medial occipital lobes. Bottom right: axial SWI MIP with near-absence of venous blood oxygen level-dependent signal in the parieto-occipital cortices.

depending on the specific imaging protocol used but always included axial diffusion, T1- and T2-weighted, fluid-attenuated inversion recovery, and susceptibility-weighted imaging or gradient recalled-echo sequences.

Cerebrovascular perfusion was evaluated by using a commercially available pulsed ASL sequence that incorporates quantitative imaging of perfusion by using a single subtraction (QUIPSS), second version (QUIPSS II), with thin-section TI, periodic saturation (Q2TIPS), and proximal inversion with a control for offresonance effects (PICORE) with the following parameters: 52 label and control image pairs with section spacing $(0-\mathrm{mm}$ section gap); $5 \mathrm{~mm}$ for 21 sections or $7.5 \mathrm{~mm}$ for 17 sections (dependent on protocol); $\mathrm{TE}=12 \mathrm{~ms} ; \mathrm{TI} 1=800 \mathrm{~ms} ; \mathrm{TI} 2=1800 \mathrm{~ms} ; \mathrm{TR}=$ $3400 \mathrm{~ms}$; receiver bandwidth $=2367 \mathrm{~Hz} / \mathrm{px}$; flip angle $=90^{\circ}$; $\mathrm{FOV}=192 ; 64 \times 64$ matrix. Quantitative $\mathrm{CBF}$ was calculated from ASL data on a voxelwise basis (in milliliters per 100 $\mathrm{g} / \mathrm{min}) .{ }^{11}$ Areas of regional hyperintensity on quantitative CBF maps (suggesting regional hyperperfusion) were visually assessed by 1 reader (J.B.A.) who was blinded to patient clinical history and outcome. Reader assessment was supplemented with ROI placement within the cortical and basal ganglia gray matter. Regional increases in CBF of $>20 \%$ (relative to global CBF) and/or $>100 \mathrm{~mL} /$ $100 \mathrm{~g} / \mathrm{min}$ were considered meaningful and defined as hyperperfusion.

After several cases demonstrated hyperperfusion predominantly in the medial occipital lobes, classified as medial occipital lobe hyperperfusion (MOLH), we differentiated MOLH and hyperperfusion in any other vascular distribution, classified as "nondistinct." Additional assessment was performed to determine the extent of diffusion restriction and abnormalities on SWI. Patient chart review was performed (A.d.H) to determine clinical outcome, which was defined as a dichotomous outcome of death or survival before hospital discharge. Descriptive and frequency statistical analyses were obtained by using STATA software, Version 13.1 (StataCorp, College Station, Texas). Statistical significance for intergroup differences was assessed by the Pearson $\chi^{2}$ or the Fisher exact test for categoric variables and by the Student $t$ or Mann-Whitney $U$ test for continuous variables.

Twenty-two patients met the inclusion criteria. Mean age was $51.0 \pm 19.6$ years with a male predominance (77.3\%). The mean admission Glasgow Coma Scale score was $3.5 \pm 1.1$, and the time from hospital admission to MR imaging was $3.2 \pm 1.7$ days. The etiology of HIE included cardiac arrhythmia and/or arrest (45.5\%), respiratory arrest and/or depression $(22.7 \%)$, drug overdose (18.2\%), and hanging (13.6\%). Eleven patients (50\%) underwent therapeutic hypothermia $\left(32^{\circ}-34^{\circ} \mathrm{C}\right.$ for $24-72$ hours). Seventeen patients demonstrated nondistinct regional hyperperfusion; 16 died. Eighteen patients had restricted diffusion of the cortical gray matter and/or the basal ganglia, and 17 died. Most patients died secondary to withdrawal of care based on the severity of their injury and poor prognostic indicators. Four of the 5 patients with normal perfusion survived. By visual inspection, the ASL abnormalities comprised smaller volumes than the diffusion abnormalities.

Eleven of 22 patients had MOLH: prominent or isolated hyperperfusion on ASL involving the bilateral medial occipital lobes (Fig 1). The presence of hyperperfusion, either in any vascular territory (nondistinct) or as MOLH, was associated with in-hospital death (nondistinct, $P=.003$; MOLH, $P=.018$ ). The presence of cortical and/or deep gray matter restricted diffusion was 


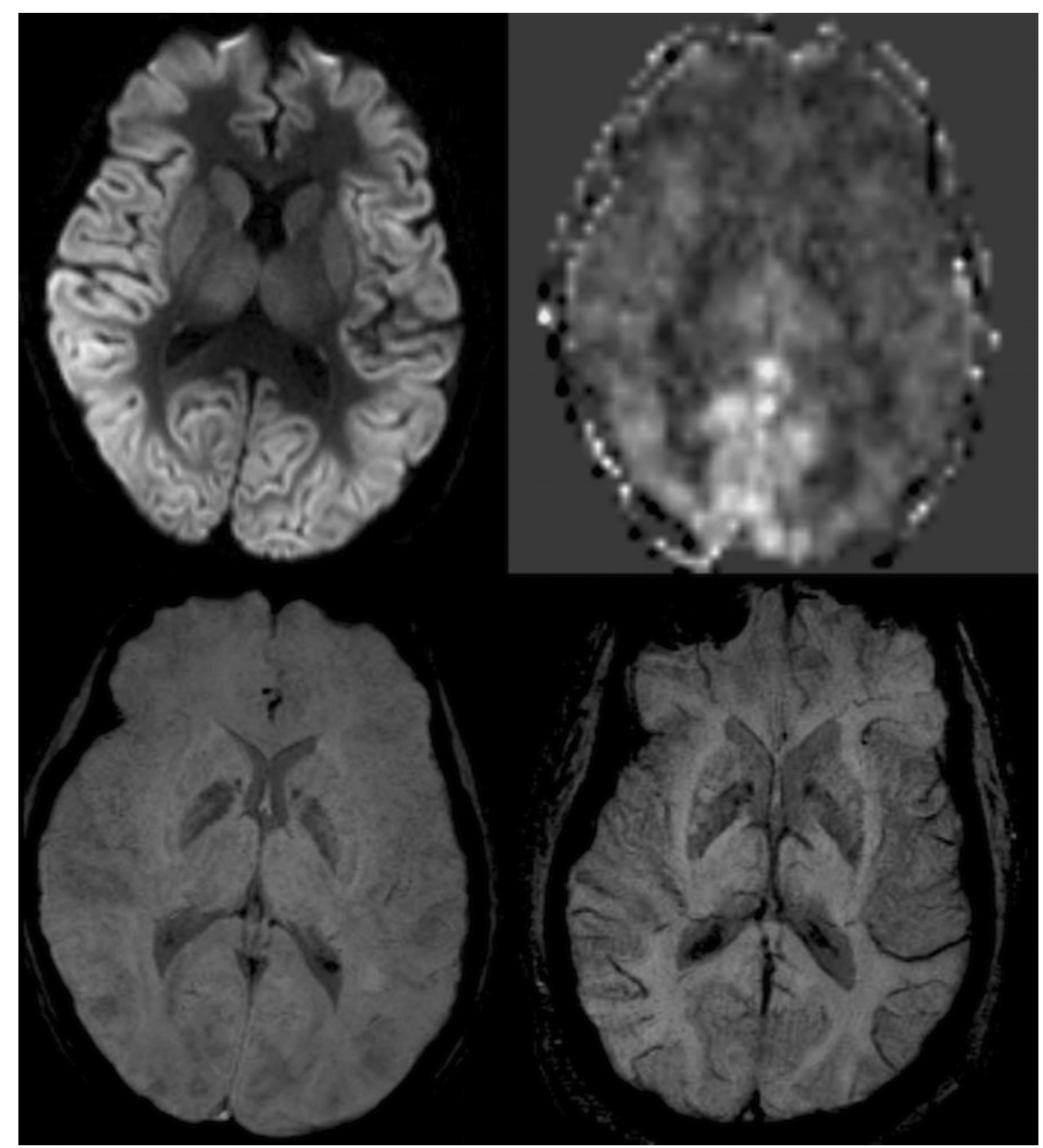

FIG 2. Brain MR imaging of a 36-year-old woman who had respiratory arrest secondary to opiate overdose 2 days prior. Top left: axial DWI with global cortical and deep gray matter diffusion restriction. Top right: axial ASL CBF with MOLH. Bottom left: axial SWI MIP with near-absence of venous blood oxygen level-dependent signal throughout the brain. Bottom right: axial SWI MIP from another patient in the cohort, for comparison, who did not have diffusion restriction or hyperperfusion, with ample venous blood oxygen level-dependent signal in both hemispheres.

specific pattern of MOLH in half of them (50\%). The mechanism of hyperperfusion following HIE is not clearly understood but is theorized to result from a delayed loss of vascular resistance following an initial period of hypoperfusion. ${ }^{6}$ The mean time to MR imaging from the hypoxic or anoxic insult was 3.2 days in our cohort, which exceeds the 24- to 48-hour period of hypoperfusion following HIE. ${ }^{4}$ The preferential involvement of the medial occipital lobes in HIE is incompletely understood but may be due to the high baseline metabolic demand of this region, rendering it more susceptible to hypoxic or anoxic injury. ${ }^{12}$

ASL hyperperfusion following HIE has been previously described, ${ }^{6,7}$ but selective hyperperfusion involving the medial occipital lobes represents a novel finding. Preferential or isolated restricted diffusion involving the occipital lobes has been reported following HIE, though we did not observe such preferential regional cortical involvement in our patient cohort. ${ }^{13}$ An interesting finding was the absence of venous deoxyhemoglobin blood oxygen level-dependent signal on SWI in areas of hyperperfusion. Following cardiac arrest, evaluation with PET has demonstrated a reduction of oxygen metabolism and extraction in the occipital lobes, associated with worse outcome. ${ }^{14}$ The specific absence of venous deoxyhemoglobin in the medial occipital lobes on minimum-

also associated with in-hospital death $(P=.001)$. Seven of the 11 patients with MOLH were treated with therapeutic hypothermia (63.6\%), while 4 of the 11 patients without MOLH (36.4\%) underwent therapeutic hypothermia $(P=.197)$. The patients with MOLH and therapeutic hypothermia were more likely to die $(100 \%, 7 / 7)$ than the patients with therapeutic hypothermia without MOLH $(25 \%, 1 / 4)(P=.024)$. Fifteen patients underwent SWI, of whom 8 demonstrated a relative absence of the typical cortical blood oxygen level-dependent signal from venous structures, which was notable in corresponding areas of hyperperfusion or diffusion restriction (Fig 2) and was associated with inpatient death $(P=.007)$. In patients with MOLH, the absent SWI blood oxygen level-dependent venous signal was identified in the occipital lobes in $4 / 4$.

\section{DISCUSSION}

Hypoxic-ischemic encephalopathy is often associated with a grim, yet uncertain, prognosis. We evaluated the presence of regional hyperperfusion in a sample of patients with severe HIE, who subsequently underwent MR imaging with ASL, and identified nondistinct regional hyperperfusion in most (77\%) patients and a intensity-projection SWI-processed images, observed in the patients with MOLH, would support this observation and suggests the development of possible physiologic shunting of diamagnetic oxygenated blood through dilated vascular channels.

We acknowledge referral bias in this study, because MR imaging examinations were preferentially ordered for patients presumed to have a poor prognosis. As a result, the mortality rate of $77.3 \%$ in our sample was notably high for HIE, but it proffered a unique opportunity to identify imaging biomarkers that could be studied in more heterogeneous cohorts of HIE. We did observe survival in 1 patient, who exhibited both deep gray matter restricted diffusion and nondistinct hyperperfusion, which are concerning false-positive findings. Nonetheless, the universal poor prognosis of patients with the highly distinctive imaging pattern of MOLH emerged from our retrospective investigation and can be viewed as hypothesis-generating.

\section{CONCLUSIONS}

ASL may have a unique complementary role in the evaluation of patients with HIE, who are often unable to undergo contrast- 
based perfusion scans secondary to acute renal insufficiency. In our small cohort, a patient survived despite having poor prognostic indicators, including nondistinct hyperperfusion and restricted diffusion. Multimodal, accurate diagnostic testing is crucial for decision-making in this setting. MOLH is a distinctive imaging pattern with a plausible physiologic basis. Our findings warrant additional study, particularly in light of the potential utility of MOLH in patients undergoing therapeutic hypothermia, which has led to uncertainty in the reliability of traditional prognostic markers validated in the prehypothermia era. ${ }^{13} \mathrm{~A}$ prospective trial would determine if these findings hold true for patients with moderate HIE, both with and without therapeutic hypothermia.

Disclosures: Jalal B. Andre-UNRELATED: Grants/Grants Pending: Philips Healthcare, ${ }^{*}$ Comments: Grant funding was provided to offset costs of MR imaging scans and related research personnel. *Money paid to the institution.

\section{REFERENCES}

1. Howard RS, Holmes PA, Koutroumanidis MA. Hypoxic-ischaemic brain injury. Pract Neurol 2011;11:4-18 CrossRef Medline

2. Greer DM, Rosenthal ES, Wu O. Neuroprognostication of hypoxicischaemic coma in the therapeutic hypothermia era. Nat Rev Neurol 2014;10:190-203 CrossRef Medline

3. Howard RS, Holmes PA, Siddiqui A, et al. Hypoxic-ischaemic brain injury: imaging and neurophysiology abnormalities related to outcome. QJM 2012;105:551-61 CrossRef Medline

4. Inoue $\mathrm{Y}$, Shiozaki T, Irisawa T, et al. Acute cerebral blood flow variations after human cardiac arrest assessed by stable xenon en- hanced computed tomography. Curr Neurovasc Res 2007;4:49-54 CrossRef Medline

5. Cohan SL, Mun SK, Petite J, et al. Cerebral blood flow in humans following resuscitation from cardiac arrest. Stroke 1989;20:761-65 CrossRef Medline

6. Pollock JM, Whitlow CT, Deibler AR, et al. Anoxic injury-associated cerebral hyperperfusion identified with arterial spin-labeled MR imaging. AJNR Am J Neuroradiol 2008;29:1302-07 CrossRef Medline

7. Wintermark P, Hansen A, Gregas MC, et al. Brain perfusion in asphyxiated newborns treated with therapeutic hypothermia. AJNR Am J Neuroradiol 2011;32:2023-29 CrossRef Medline

8. Deibler AR, Pollock JM, Kraft RA, et al. Arterial spin-labeling in routine clinical practice, Part 1: technique and artifacts. AJNR AmJ Neuroradiol 2008;29:1228-34 CrossRef Medline

9. Eckardt KU, Bernhardt WM, Weidemann A, et al. Role of hypoxia in the pathogenesis of renal disease. Kidney Int Suppl 2005:S46-51 CrossRef Medline

10. Devarajan P. Update on mechanisms of ischemic acute kidney injury. J Am Soc Nephrol 2006;17:1503-20 CrossRef Medline

11. Wang J, Licht DJ, Jahng GH, et al. Pediatric perfusion imaging using pulsed arterial spin labeling. J Magn Reson Imaging 2003;18:404-13 CrossRef Medline

12. Rabinstein A, Resnick SJ. Hypoxic-ischemic brain damage. In: Rabinstein A, Resnick SJ. Practical Neuroimaging in Stroke: A Case-Based Approach. Philadelphia: Saunders; 2009:1-17

13. Hahn DK, Geocadin RG, Greer DM. Quality of evidence in studies evaluating neuroimaging for neurologic prognostication in adult patients resuscitated from cardiac arrest. Resuscitation 2014;85: 165-72 CrossRef Medline

14. Edgren E, Enblad P, Grenvik A, et al. Cerebral blood flow and metabolism after cardiopulmonary resuscitation: a pathophysiologic and prognostic positron emission tomography pilot study. Resuscitation 2003;57:161-70 CrossRef Medline 